\title{
Nursing Education Intervention Effects on Pain Intensity of Nursing Home Residents with Different Levels of Cognitive Impairment: A Cluster-Randomized Controlled Trial
}

This article was published in the following Dove Press journal:

Journal of Pain Research

\section{P Kutschar (D) ${ }^{\prime}$ \\ S Berger' \\ A Brandauer (D) \\ N Freywald (D) ${ }^{\prime}$ \\ J Osterbrink ${ }^{1,2}$ \\ D Seidenspinner ${ }^{3}$ \\ I Gnass'}

'Institute of Nursing Science and Practice, Paracelsus Medical University, Salzburg, Austria; ${ }^{2}$ Brooks College of Health, University of North Florida, Jacksonville, FL, USA; ${ }^{3}$ Nursing Science and Practice Development, University Hospital of Munich-Großhadern (LMU), Munich, Germany
Correspondence: P Kutschar

Institute of Nursing Science and Practice,

Paracelsus Medical University,

Strubergasse 21, Salzburg 5020, Austria

$\mathrm{Tel}+4366280344$

Email patrick.kutschar@pmu.ac.at
Purpose: Pain management in nursing homes is challenging and pain prevalence remains high. The objective of this study was to improve the pain situation of nursing home residents following a nursing-related educational intervention within a cluster-randomized controlled trial (2016-2018).

Participants: Clusters were nursing homes from one nursing home operator in Bavaria, Germany. Nursing home residents who were permanently registered in the facilities, at least 60 years of age, and who themselves or their legal guardians provided informed consent were included. Intervention: In addition to the implementation of pain nurses and pain care assistants, staff of the intervention group received an educational intervention in pain management, containing classroom (quality circles) and web-based training for nurses.

Methods: Based on the Mini-Mental State Examination (MMSE), residents were either interviewed (MMSE 10-30) using self-report instruments or observed (MMSE 0-9) by proxy assessment. The primary outcome in residents able to self-report was maximum pain intensity according to Brief Pain Inventory (BPI); in those not able to self-report treatmentrelevant pain above cut-off $(\geq 2)$ on the Pain Assessment in Advanced Dementia (PAINAD). Results: Out of 20 randomly selected clusters, 9 nursing homes from the control, and 6 nursing homes from the intervention group participated. Multilevel linear $(n=347$ residents, MMSE 10-30) and logistic regression ( $n=222$ residents, MMSE 0-9) analyses were conducted. Maximum pain intensity was higher after intervention $(B=1.32, p<0.01)$, decreased with a better quality of life $(B=-0.07, \mathrm{p}<0.001)$, and was lower when dementia diagnoses were present $(\mathrm{B}=-1.12, \mathrm{p}<0.01)$. PAINAD scores before and after intervention did not differ significantly $(\mathrm{OR}=0.89, \mathrm{p}=0.724)$, but chances to exhibit treatment-related pain were higher with decreasing MMSE $(\mathrm{OR}=0.94, \mathrm{p}<0.05)$.

Conclusion: While no significant positive intervention effect was measured, findings suggest nurses' raised awareness towards pain management. Overall results indicate that large-scale educational interventions seem to be less effective in complex nursing home settings without also including specific individual-based intervention measures.

Keywords: nursing home residents, pain management, nurse-related intervention, nursing education, cognitive impairment, dementia

\section{Introduction}

The continuously high prevalence of acute and chronic pain in nursing home residents (NHR) represents a critical challenge in nursing and medical care. ${ }^{1-3}$ Although 
the estimates of pain prevalence vary due to different study procedures or pain measures, international studies suggest that up to four out of five residents experience pain regularly. ${ }^{4-7}$ High pain prevalence, typically caused by musculoskeletal, cardio-/vascular, neurological disorders and cancer, has been found to be associated with decreasing cognitive abilities, more pronounced anxiety or malnutrition, higher levels of severe depression, multi-morbidity, or more distinct behavioral disturbances. Overall, being affected by pain poses a significant burden with farreaching consequences for social interaction, activities of daily living, and quality of life..$^{8-12}$

Progressed cognitive impairment (CI) and dementia symptoms are common causes why people move into longterm care facilities. Numbers of residents with documented dementia in nursing homes $(\mathrm{NH})$ often exceed rates of $50 \%$ and as many as $80 \%$ of the residents are assumed to be cognitively impaired. ${ }^{13,14}$ Cognitive complaints and interrelations with pain and other co-morbidities further complicate the professional care in NH. Pain management for NHR with progressing cognitive decline entails particular challenges. This becomes evident during pain assessment as the prerequisite of an adequate pain management. ${ }^{8}$ NHR with advanced CI may specifically interpret pain sensation and perception, have significant problems recalling painful situations, and may no longer be able to communicate their pain reliably. ${ }^{15-17}$ For NHR who are not able to self-report, pain assessment strategies have to draw on the usage of differentiated behavioral pain assessment tools. ${ }^{10,18}$ While NHR with up to moderate cognitive impairment are often still able to describe and report their pain verbally, this can hardly be expected from most individuals with severe CI. ${ }^{19-21} \mathrm{CI}$ may not only restrict possibilities to accurately assess pain, but also limit the scope and application of pharmacological and non-pharmacological pain treatment strategies. ${ }^{22,23}$ Hence, pain in NHR with different levels of CI remains alarmingly under-assessed and under-treated. ${ }^{8,16,24,25}$

Frequently reported care-related barriers to adequate pain management are unsatisfactory training of nurses, lacking knowledge and education about pain management measures, deficient institution-specific procedural regulations, inadequate assessment and documentation of pain, or lacking evidence-based practice skills. ${ }^{26-30}$ Nonetheless, the improvement of the NHR' pain situation should serve as an unconditional goal of pain management in order to provide the best possible quality of life, functionality, and social participation of the affected.
Meanwhile, there is an ever-growing body of research about the effectiveness of very specific pain assessment and treatment interventions on various dimensions of pain and associated phenomena for populations with and without CI. ${ }^{9,11,31-34}$ Only few studies have focused explicitly on effects of more general interventions in pain management strategies in $\mathrm{NH}$, indicating that implementing changes in pain management practices is challenging regardless of the health-care setting. ${ }^{35,36}$ Educational interventions which aim at improving nurses' knowledge and competencies in pain management are rather rare, yet some were found to have positive outcomes for individuals' pain situation. ${ }^{30,36-38}$ There is a clear need for studies to test large-scale pain management intervention effects in NH. In particular, randomized controlled trials and evidence-based studies are scarce and most warranted. ${ }^{27,36,39}$

\section{Purpose}

The primary goal of the PIASMA study was to improve the residents' pain situation following the implementation of specific nursing-related interventions. It was assumed that after the implementation of an educational intervention package for nurses, the residents in the intervention group will present with an improved pain situation. NHR with all levels of cognitive states were included in order to avoid a systematic exclusion due to impairment in cognitive function.

Anticipating the effectiveness of the implemented intervention, it was hypothesized that

(a) the average maximum pain intensity in residents able to perform self-assessment (no, mild, moderate $\mathrm{CI}$ ) in the intervention group will be significantly lower than in the control group and

(b) the proportion of treatment-relevant pain indicators of residents unable to perform self-assessment (severe CI) in the intervention group will be significantly lower than in the control group.

\section{Materials and Methods}

\section{Study Design}

A cluster-randomized controlled trial (cRCT) was conducted to evaluate the effects of a nursing education intervention on NHR' pain intensity and presence. NH were defined as the cluster-level as it is hardly possible to control contamination across residents in case of individual randomization and intervention provision strategies. Variation in structures, procedures or pain management provision between NH tends to result in differences between but homogeneities within 
clusters and cRCT methodology qualifies to account for these situations. Between baseline (2016-2017) and followup (2017-2018), the intervention phase lasted 12 months. Interventions took place at the cluster-level and outcomes were measured at the individual NHR level. This WHOregistered trial (UTN: U1111-1187-3174) was conducted in $\mathrm{NH}$ of one single nursing home operator in the German federal state of Bavaria, Germany.

\section{Intervention}

During the intervention phase, an educational intervention package was offered and implemented in collaboration with the quality and nursing home managers in charge from the intervention group. All components of the package and each training program were developed according to the pain curriculum of the German Pain Society. ${ }^{40}$ Nurses with different qualification levels (ie registered and non-registered nurses) were eligible for the intervention. Furthermore, for the implementation of the gained knowledge and skills in pain management nursing, certain tasks and roles were given to the participants of the different training programs. The package involved:

(a) Advanced training for select nurses (registered) as certified pain nurses (distance learning program; three topical modules 'basic', 'acute', and 'chronic pain management'; elaboration on five training letters and exercises over a 150-day-course timeframe; lectures about primary aspects of pain management nursing according to up-to-date guidelines and frameworks). In each NH in the intervention group, at least one registered nurse was selected for training and for leading the intervention phase to support the optimization of pain management nursing within their $\mathrm{NH}$, which was also implemented into a newly established job description.

(b) Advanced training for select nurses as certified pain care assistants (presence learning program; identification of supportive responsibilities in pain management; training on pain management basics primarily focusing on the understanding and recognition of pain and painful situations, the basics in pharmacological and non-pharmacological treatment, and patient education and interdisciplinary communication; in total 32 classroom sessions of 45 minutes each). In each $\mathrm{NH}$ in the intervention group, at least two nurses (registered and unregistered) per ward were intended for training and to support the dissemination of knowledge and skills throughout the nursing team.

(c) Web-based training video on chronic pain and care of persons with dementia for all nursing staff (selflearning course; four topical online-videos lasting between 7 and 14 minutes; contents comprised causes of pain in NHR with dementia, usage of proxy assessment instruments, ie PAINAD, and its importance for everyday care procedures, video examples of simulated assessment situations and structured feedback). The access to the web-based training video was distributed through the nursing home manager and could be performed at computers in the NH. Reminders for participation within the intervention phase were announced twice.

(d) Implementation of interdisciplinary quality circles (two whole-day classroom meetings at $\mathrm{NH}$ site conducted by the research team; participants were superordinate quality managers, pain nurses-in-training and nurses in charge of the implementation of trials' interventions; contents included training and exercises on pain assessment procedures for residents able and not able to self-report, instruction on how to implement case reports on regular bases, instructions regarding pain treatment protocols according to the German national standard of pain management in nursing). ${ }^{41}$

No intervention was applied in the nursing homes belonging to the control group. Participation, implementation and degree of intervention penetration varied between the clusters belonging to the intervention group. Overall, three specialized pain nurses and 15 pain care assistants were trained. The small number of nurses who took part in the training was reasoned by the $\mathrm{NH}$ managers insofar as it was challenging to find nurses willing to participate in the training and be in charge of the new role. Nursing staff from all NH participated in the online education tool for pain assessment in patients with cognitive impairment. All designated (pain) nurses participated in the quality circle at least once. Overall, the intervention package enabled all nurses to assess pain based on the NHR' individual degree of CI, to administer pharmacological pain treatment as prescribed and to ensure further non-pharmacological nursing measures. Additionally, the nurses were qualified to give the relevant information on pain treatment to the NHR and their legal guardians and - if applicable - to provide advanced pain education.

In order to be analyzed as intervention group $\mathrm{NH}$ after follow-up, at least one pain nurse or pain care assistant had 
to be implemented at site as well as at least one quality circle had to take place. These criteria could not be fulfilled by one $\mathrm{NH}$ due to staff turnover and lacking resources which was communicated by the NH manager at the beginning of the intervention phase. This particular $\mathrm{NH}$ was allocated to the control group as no intervention measures were delivered.

\section{Randomization Procedure and Blinding}

The total number of eligible clusters was $42 \mathrm{NH}$, of which 39 $\mathrm{NH}$ met the inclusion criteria. A simple random urn (bowl drawing without replacement) sampling procedure was applied in July 2016 to select $20 \mathrm{NH}$ and the two-arm parallel allocation ratio was 1:1. Hence, out of 20 selected $\mathrm{NH}, 10 \mathrm{NH}$ were allocated to the intervention group and $10 \mathrm{NH}$ to the control group at the second-stage randomization. Randomization was performed by the research team including the responsible statistician. After allocation, the nursing home operators' executives and internal project coordinator informed all nursing home managers to ensure motivation, cooperation and commitment. Detailed written project information was given to all $\mathrm{NH}$ managers and to the designated study nurses in each $\mathrm{NH}$.

NHR, their legal guardians, and raters (ie interviewers) were blinded to the allocation for both baseline and followup. Control and intervention allocation were blinded for $\mathrm{NH}$ managers and NH staff at baseline, but not at follow-up as the allocation necessarily became obvious due to the provision of staff education during the intervention phase. Nursing home managers, study nurses and nurses, who participated in the intervention program, were asked to strictly conceal all information about intervention or control allocation from residents, legal guardians, or raters.

\section{Participants and Inclusion Criteria}

The inclusion criteria at the $\mathrm{NH}$ level was a minimum occupancy of 50, exclusive of special care services (eg care of NHR with psychiatric disorders). Also, facilities with assisted living care or sheltered housing were excluded.

Eligible participants at the individual level were all NHR from the participating $\mathrm{NH}$ who were permanently registered in the facilities, being $\geq 60$ years of age, and provided informed consent by themselves or by their legal guardian. $\mathrm{NH}$ managers and ward nurses were trained in the inclusion and exclusion criteria and provided a list of eligible participants. Residents in short-term care or day-care, suffering from congenital/ permanent multiple disabilities, finding themselves in life-threatening situations, or persons with an insufficient command of the German language were excluded.

NHR were stratified into two groups of cognitive impairment using the Mini-Mental State Examination (MMSE). ${ }^{42,43}$ The MMSE estimates cognitive function on five dimensions relating to orientation ability, memory performance, comprehension capability, visual construction and language usage. The MMSE results in a score ranging from 0 to 30 points with lower scores indicating more severe impairments. In order to maintain the autonomy and independence of residents with $\mathrm{CI}$, an inclusive strategy in determining ability to interview was pursued: NHR with up to moderate impairment (MMSE 10-30) were interviewed with questionnaires, residents with severe impairments (MMSE 0-9) were examined using behavioral (proxy) assessment instruments. ${ }^{19,21,44}$ Regarding the self-report group, our study assistants (ie interviewer, rater) were trained to abort the interview in case residents showed conspicuous response behavior (eg obviously not being able to understand questions, not able to convey an adequate answer or providing completely out-ofcontext responses). Hence, in case self-report turned out to not be feasible during assessment, NHR were assigned to the proxy assessment group regardless of the previously measured MMSE score.

\section{Data Collection Procedures}

Next to a general online CASI-survey of certified nurses (data not presented as the survey was not part of the RCT framework), structural data on aggregated regulations and procedures were gathered, resident-specific care records and medical characteristics were collected, and NHR were examined according to their cognitive skills (ie selfreport or proxy assessment). Structural data and resident characteristics were derived from the NH operators' centralized electronic care record system under the supervision of the IT managers in strict compliance with data privacy regulations. Data were already pseudonymized at this stage.

The fieldwork per NH lasted 5 days on average. Data collection was carried out by interviewers who were either students of health sciences, health-care professionals or selected nurses of the included nursing homes. All interviewers participated in a comprehensive interviewer training and were supervised by the research team during data collection. In the sample of NHR who were supposed to be able to self-report on their pain situation, a strict standardized survey approach was pursued. Questions were read aloud from survey tablets and additionally presented to the 
NHR visually as print versions. NHR with severe CI were examined using standardized proxy assessment instruments. Interviewers observed select pain-related care situations and assessed pre-specified pain criteria. In addition, the responsible nurses rated the occurrence of neuropsychiatric symptoms for each individual NHR.

\section{Measurements and Outcomes Nursing Home-Level}

The nursing home-level variables used to present analyses were the size of the nursing home in terms of the total number of resident occupancy, and the total number of registered nurses as well as nursing assistants.

\section{Resident-Level Primary Outcomes}

The primary outcome for NHR able to perform selfassessment was the average maximum pain intensity during the previous $24 \mathrm{hrs}$ as part of the modified and validated German version of the Brief Pain Inventory (BPI). ${ }^{45,46}$ BPI measures pain aspects along the two scales "pain intensity" and "pain interference." The pain intensity scale assesses least, maximum, average and actual pain intensity during the last $24 \mathrm{hrs}$ (four NRS-11 items). The pain interference scale comprises seven items (NRS-11) to assess the impact of pain. The outcome variable "maximum pain intensity" comprised 11 answer options from " 0 -no pain" to '10-worst pain imaginable' and was treated as a metric outcome in multilevel analyses. The primary outcome for NHR assumed to be unable to perform self-assessment was the amount of pain indicators collected by means of proxy assessment, measured according to Pain Assessment in Advanced Dementia-Scale (PAINAD). ${ }^{47}$ PAINAD is an instrument to assess relevant signs of pain while observing potentially pain-inducing situations (eg transfer, mobilization). After observing residents for several minutes, five behavior dimensions (breathing, negative vocalization, facial expression, body language, consolability) have to be scored using a standardized rating scheme. The total score ranges between 0 and 10 points with a higher score indicating more relevant behavioral signs of pain. The validated German version of PAINAD was used and a recommended cut-off ( $\geq 2$ points) for a "probable pain indication" was applied. ${ }^{48,49}$ PAINAD was treated as a dichotomous outcome variable in multilevel modelling.

\section{Resident-Level Secondary Outcomes and Controls}

In the self-report group, depression and quality of life were measured as secondary outcomes. The German version of the Geriatric Depression Scale GDS was used to estimate signs of depression. ${ }^{50,51}$ The GDS-short form consists of 15 dichotomous items indicating depressive symptoms during the last 7 days, each item equally contributing to a total score between 0 and 15 points. A score of $\geq 6$ points is recommended as cut-off indicating non-ignorable signs of depression. ${ }^{50}$ Health-related quality of life ("HRQoL") was assessed by the EQ-5D-3L, which consists of five dimensions (mobility, self-care, usual activities, pain/discomfort, anxiety/depression) with three answer levels each and a self-rating of health on a visual analogue scale. ${ }^{52}$ The ratings on the five dimensions were transformed into a sum index ranging from 0 ("worst HRQoL") to 100 ("best HRQoL") points. ${ }^{53}$

The secondary outcome for residents with severe CI was the nursing home version of the Neuropsychiatric Inventory NPI-NH. ${ }^{54}$ NPI measures the frequency and severity of neuropsychiatric symptoms for each resident based on informed nurses' ratings on 10 neuropsychiatric domains (eg delusions/hallucinations, arousal/aggression, anxiety, euphoria) and 2 neuro-vegetative indicators (sleep and nighttime behavioral disorders, changes in appetite and eating habits). Indexes can be computed for each item adding up to a total NPI index from 1 to 12 points, whereby a cut-off for clinically relevant behavior of $\geq 4$ points is established. ${ }^{54}$

For all NHR, gender, age, dementia, depression as well as pain-associated diagnoses were extracted from the centralized electronic care record system. While age in years was measured as a continuous variable, gender and present diagnoses were represented as dichotomous variables. Pain-associated diagnoses were categorized into tumor (eg colon cancer, prostate/breast cancer), musculoskeletal (eg fractures, osteoporosis) and neuropathic (eg herpes zoster, multiple sclerosis) diagnoses due to low frequencies of the specific primary diagnoses.

\section{Sample Size Calculation}

Anticipated effect sizes built up upon the IMMPACT recommendations for chronic pain clinical trials. ${ }^{55}$ Clinically relevant reduction in maximum pain intensity was set to 2 scale points on NRS (0-10); a relevant change in the proportion of PAINAD above cut-off a $20 \%$ reduction (control vs intervention group: 60 vs $40 \%$ ) was anticipated. Sample size calculation was based on t-tests for independent samples $(\beta=80 \%$, one-sided $\alpha=0.05$ ) for maximum pain intensity and z-tests for independent proportions for the PAINAD cut-off. Parameters used for sample size calculation were as follows: participation rate $=60 \%$, 
unit nonresponse $=20 \%$, administrative loss of data $=5 \%$, proportion of $\mathrm{NHR}_{\text {MMSE } 10-30}$ to $\mathrm{NHR}_{\text {MMSE } 0-9}=75: 25 \%$, intraclass correlation coefficients $(\mathrm{ICC})=0.05$, and average cluster sizes of $36.1 \mathrm{NHR}_{\text {MMSE }} \quad 10-30$ and 12.01 NHR $_{\text {MMSE }}$ 0-9. Calculated optimal sample sizes were 220.8 NHR $_{\text {MMSE } 10-30}$ and 238.7 NHR MMSE 0-9. In order to achieve these numbers, $645.6 \mathrm{NHR}_{\mathrm{MMSE}} 10-30$ and

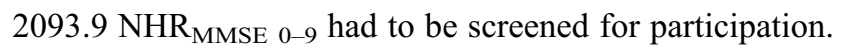
Given an average NH size of 105.68 , the number of $\mathrm{NH}$ to

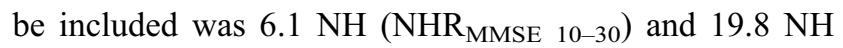
(NHR MMSE 0-9). Hence, sufficient sample size at the cluster level was set to $20 \mathrm{NH}$.

\section{Statistical Analyses}

Common descriptive statistics were used to characterize baseline data. To explore the effect of the educational interventions adjusted by secondary outcomes and controls, and to take possible variation due to clustering into account, multilevel analyses with NHR (level 1) nested in $\mathrm{NH}$ (level 2) were modelled. Independent sample data were used for multilevel modelling. For the continuous outcome "maximum pain", two-level linear regression models (restricted maximum likelihood method) were applied. For the binary outcome "PAINAD above cut-off", two-level logistic regression models (robust covariances method) were applied. For both subsamples, several models were examined, starting with the null model only including the random intercept and successively adding independent variables to identify variables significantly associated with the outcome and explaining the variation on different levels whilst adjusting stepwise:

(a) Null model: random intercept model

(b) Model 1: random intercept model including intervention (time $\times$ group interaction) variable

(c) Model 2: random intercept model, additionally adjusting for resident characteristics

(d) Model 3: random intercept model, additionally adjusting for resident-level secondary outcomes

(e) Model 4: random intercept model, additionally adjusting for nursing home-level characteristics

The time $\times$ group interaction term (ie significant coefficients representing an intervention effect) and all independent variables were included as fixed effects. The full model 4 incorporating level 1 and level 2-variables was used for the final interpretation of results. The results for the null and full model only are presented (see Supplementary materials for full details). In order to facilitate the interpretation of intercepts, grand mean centering was applied for resident-level variables, where applicable. ICC was calculated for each model. All multilevel analyses were conducted in IBM SPSS Statistics 24.0. Data analyses followed the as treated principle. A sensitivity analysis using the intention-to-treat principle and analyzing the clusters as randomized was performed.

\section{Ethical Clearance}

The responsible ethics committee of the medical faculty of Ludwig Maximilian University Munich (Bavaria, Germany) gave ethical clearance and approved the study protocol and procedures (ref.: 379-16). Participants - or legal guardians of participants - provided written informed consent, which covered information about the study procedures, data confidentiality, anonymity and participants' unconditional right to withdraw from the study at any time. Personal data of residents were pseudonymized at the $\mathrm{NH}$ site and strictly separated from analysis at all times. This cRCT was conducted in accordance with the Declaration of Helsinki.

\section{Results}

\section{Enrolment, Allocation, and Participants}

In total, $42 \mathrm{NH}$ with $3990 \mathrm{NHR}$ were assessed for eligibility. Thirty-nine NH with 3845 NHR corresponded to the clusterlevel inclusion criteria. Twenty NH were randomly selected and equally allocated either to the intervention or control group. During baseline data collection, $3 \mathrm{NH}$ from the intervention, and $2 \mathrm{NH}$ from the control group dropped out from the study due to nursing home managers' reasoned noncompliance or extremely low NHR' expression of interest rates to participate. In all, 177 NHR from 7 NH (allocation: intervention) and $332 \mathrm{NHR}$ from $8 \mathrm{NH}$ (allocation: control) were examined at baseline (t0). After transferring one $\mathrm{NH}$ from intervention to control group due to the lack of any implemented intervention, 176 NHR from intervention group $\mathrm{NH}$ and 196 NHR from control group NH were examined at 12month follow-up (t1). For statistical modelling, data were prepared as independent samples; in all, 714 NHR from 15 $\mathrm{NH}$ were available for analysis. Figure 1 illustrates the details of enrolment, allocation and recruitment procedures for clusters and individuals throughout the cRCT.

\section{Baseline Characteristics}

At the cluster-level, the average number of care places (occupancy) was distinctly higher in the $\mathrm{NH}$ allocated to 


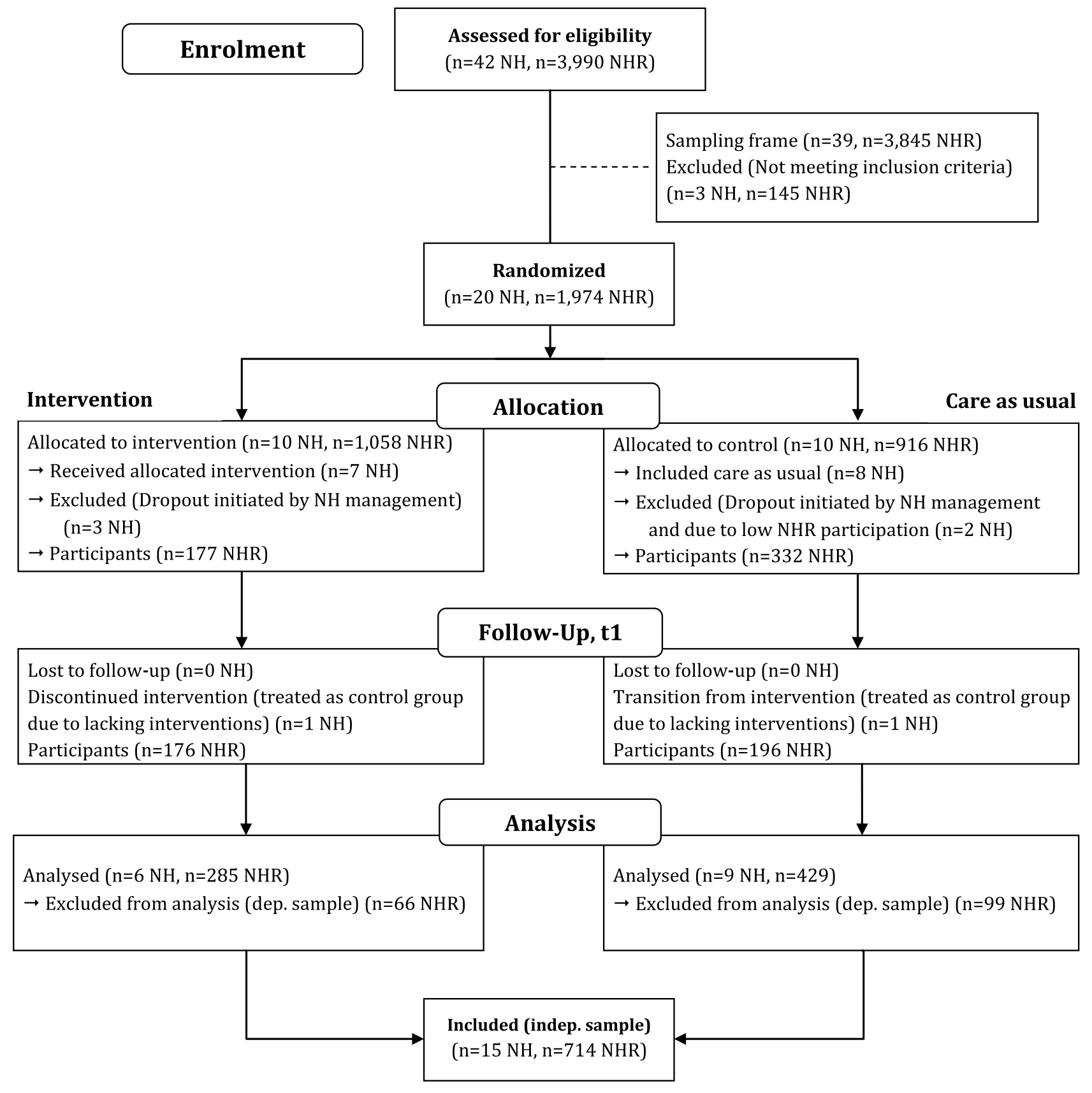

Figure I Flow diagram for enrolment, allocation and analysis.

the control than intervention group (113.3 vs 135.0 care places), which is also indicated by higher mean numbers of registered (23.3 vs 25.2 nurses) and assistant nurses (23.7 vs 28.3 nurses). The participants were stratified based on the MMSE score results into 234 NHR with no, mild or moderate CI and 108 NHR with severe CI, representing a 69:31\%-ratio. In the former subsample, most variables were similarly distributed between intervention and controls. Around two-thirds of participants were women, the mean age was 82 years and an average
MMSE scored 21 points. While the mean number of painassociated diagnoses was almost equal (1.3 diagnoses), obvious differences in proportions were observed only for neuropathic (6.1 vs 13.9\%) and tumor diagnoses (22.7 vs 9.7\%). Primary and secondary outcome distributions are comparable between intervention and control group residents. Mean maximum pain was around 2.8 points on the 11-point NRS at baseline, BPI pain intensity score based on four pain measures averaged at 1.5 and 1.8 points, respectively. Pain (at least mild pain) was present 
in approx. $60 \%$ of all NHR with up to moderate CI. More distinct interference on everyday life activities due to pain was reported in the intervention group. The mean scores of GDS ( $\mathrm{AM}=5.2$ vs 5.5; 38 vs $44 \%$ above cut-off) and EQ-5D (65 vs 67 points) were similar based on descriptive statistics. Some differences between intervention and control group participants are rather obvious in the NHR subsample with severe CI. While $69 \%$ of the residents in the intervention group were female, this holds true for only $41 \%$ in the controls. They were comparable in terms of age ( $\mathrm{AM}=82$ vs 84 years), MMSE (AM 2.2 vs 2.9 points), and the mean number of pain-associated diagnoses (1.5 vs 1.3 diagnoses). About four out of five residents were living with a dementia diagnosis in both intervention and control. In the controls, prevalence of diagnosed depression (10.3 vs $18.2 \%)$ and neuropathies (0.0 vs $3.0 \%)$ were higher, those of musculoskeletal (35.9 vs $21.2 \%$ ) and tumor (23.1 vs $10.6 \%$ ) diagnoses were lower as compared to the intervention group. Details of baseline descriptive statistics at the resident and nursing home-level included in the statistical models are displayed in Table 1.

\section{Intervention Effects on Primary Pain Outcomes}

Multilevel analyses were applied to examine a change in primary pain outcomes due to intervention, to identify significantly associated variables at the resident and nursing home-level, and to estimate how much variation in pain outcomes is attributable to nursing home-level factors.

Table I Resident and Nursing Home Level Baseline-Independent Sample Characteristics

\begin{tabular}{|c|c|c|c|c|}
\hline \multirow[t]{2}{*}{ Resident-Level } & \multicolumn{2}{|l|}{ MMSE I0-30 } & \multicolumn{2}{|l|}{ MMSE 0-9 } \\
\hline & Intervention & Control & Intervention & Control \\
\hline Female \% & 65.2 & 70.3 & 69.2 & 40.9 \\
\hline Age in years & 81.68 (10.03), 60-98 & 82.55 (8.96), 60-100 & 82.46 (9.20), 60-99 & 84.38 (7.64), 65-99 \\
\hline MMSE score ${ }^{a}$ & $20.4 I(5.65), 10-30$ & 20.53 (5.74), 10-30 & $2.18(3.10), 0-9$ & $2.87(3.18), 0-9$ \\
\hline Pain-associated diagnoses & 1.29 (0.97), 0-4 & $1.28(1.11), 0-5$ & 1.50 (0.82), , $0-4$ & I.3। (0.76), 0-3 \\
\hline Dementia \% & 42.4 & 37.6 & 82.1 & 77.3 \\
\hline Depression \% & 16.9 & 20.0 & 10.3 & 18.2 \\
\hline Musculoskeletal \% & 31.8 & 34.5 & 35.9 & 21.2 \\
\hline Neuropathies \% & 6.1 & 13.9 & 0.0 & 3.0 \\
\hline Tumor \% & 22.7 & 9.7 & $23.1 \% *$ & $10.6 \% *$ \\
\hline BPI maximum pain ${ }^{\mathrm{b}}$ & $2.78(3.61), 0-10$ & 2.81 (3.48), $0-10$ & - & - \\
\hline BPI pain intensity score ${ }^{b}$ & $1.47(2.46), 0-10$ & 1.77 (2.42), 0-8.25 & - & - \\
\hline BPI pain interference score ${ }^{c}$ & 36.49 (19.14), 0-70 & 28.95 (16.46), 0-66 & - & - \\
\hline BPI pain presence $\%$ & 58.0 & 61.8 & - & - \\
\hline GDS- $15^{d}$ & 5.22 (3.60), $0-13.85$ & 5.54 (3.49), 0-13.75 & - & - \\
\hline GDS-15 cut-off $(\geq 6) \%$ & 38.1 & 44.0 & - & - \\
\hline$E Q-5 D^{e}$ & $65.24(23.61), 20-100$ & 67.31 (20.45), 20-100 & - & - \\
\hline PAINAD $^{f}$ & - & - & $3.38(2.56), 0-8$ & $2.06(2.13), 0-7$ \\
\hline PAINAD cut-off $(\geq 2) \%$ & - & - & 67.6 & 49.3 \\
\hline$N P I^{g}$ & - & - & $5.14(2.71), 0-12$ & 4.24 (2.3I), $0-11$ \\
\hline NPI cut-off $(\geq 3) \%$ & - & - & 68.6 & 62.5 \\
\hline Number of participants & 69 & 165 & 40 & 68 \\
\hline Nursing Home-level & \multicolumn{2}{|l|}{ Intervention } & \multicolumn{2}{|l|}{ Control } \\
\hline Size in terms of care places & \multirow{3}{*}{\multicolumn{2}{|c|}{$\begin{array}{l}\text { II } 3.33 \text { (43.54), 50-154 } \\
23.33 \text { (10.89), 10-43 } \\
23.67 \text { (8.78), 10-34 }\end{array}$}} & \multirow{3}{*}{\multicolumn{2}{|c|}{$\begin{array}{l}\mid 35.00(30.4 \mid), 80-166 \\
25.22(\mid 0.39),|0-4| \\
28.33 \text { (|0.24), 9-45 }\end{array}$}} \\
\hline Number of registered nurses & & & & \\
\hline Number of nursing assistants & & & & \\
\hline
\end{tabular}

Notes: Mean (standard deviation), minimum-maximum are displayed for continuous variables, \% valid percentage for categorical variables, - not applicable. ${ }^{\mathrm{a}}$ range $0-30$ (lower scores indicate more progressed cognitive decline), ${ }^{b}$ range 0-10 (higher scores indicate more pain intensity), 'range 0-70 (higher scores indicate more interference), ${ }^{\mathrm{C}}$ range 0-15 (higher scores indicate more signs of depression), ${ }^{\mathrm{e}}$ range $0-100$ (higher scores indicate better quality of life), ${ }^{f}$ range $0-10$ (higher scores indicate more signs of pain), ${ }^{g}$ range $1-12$ (higher scores indicate more relevant neuropsychiatric behaviors); *Significant difference between control and intervention group $(p<0.05)$.

Abbreviations: MMSE, Mini-Mental Status Examination; BPI, Brief Pain Inventory; GDS, Geriatric Depression Scale; EQ-5D, Euroqol Quality of Life; PAINAD, Pain Assessment in Advanced Dementia Scale; NPI, Neuropsychiatric Inventory. 


\section{Residents with No, Mild and Moderate Cognitive Impairment}

Table 2 presents the results of linear regression multilevel analysis predicting the maximum pain intensity in NHR with up to moderate CI. The final model $(n=347)$ adjusted by resident and nursing home-level characteristics demonstrated a significant change $(\mathrm{B}=1.32, \mathrm{SE}=0.42, \mathrm{p}=0.006)$ in BPI maximum pain intensity due to time $\times$ group interaction (aka intervention effect), indicating that the mean pain intensity increased around one NRS-category. Further variables were found to be significantly correlated with NHR' maximum pain intensity. If a dementia diagnosis was present, pain intensity scores were significantly lower
$(\mathrm{B}=-1.12, \mathrm{SE}=0.42, \mathrm{p}=0.008)$. Better quality of life was significantly associated with lower maximum pain intensity scores $(\mathrm{B}=-0.07, \mathrm{SE}=0.01 ; \mathrm{p}<0.001)$. Parameter estimates of age, gender and the cognitive status of residents did not significantly predict pain intensity. One level 2-variable was associated with the intensity of maximum pain and significant by trend $(p<0.10)$ : With increasing numbers of registered nurses lower pain intensity scores were observed $(\mathrm{B}=-0.07, \mathrm{SE}=0.04, \mathrm{p}=0.076)$. The vast majority of the variance was found at the resident-level (approx. 97\%).Intraclass correlation coefficients were rather low $\quad\left(\mathrm{ICC}_{\min }=0.9 \%, \quad \mathrm{ICC}_{\max }=3.8 \%, \quad \mathrm{ICC}_{\text {fullmodel }}\right.$ $=3.0 \%$ ) in all models.

Table 2 Multilevel Linear Regression Model Predicting BPI Maximum Pain (MMSE 10-30)

\begin{tabular}{|c|c|c|c|c|}
\hline \multirow{2}{*}{$\begin{array}{l}\text { Random Intercept Linear Regression Multilevel Model } \\
\text { for Maximum Pain }\end{array}$} & \multicolumn{2}{|l|}{ Null Model } & \multicolumn{2}{|c|}{ Resident and Nursing Home-Level Model } \\
\hline & Estimate (SE) & $\mathbf{p}$ & Estimate (SE) & $\mathbf{p}$ \\
\hline $\begin{array}{l}\text { Fixed model part }{ }^{b} \\
\text { Intercept } \\
\text { Time } \times \text { group }\end{array}$ & $3.20(0.22)$ & $<0.001$ & $\begin{array}{l}3.55(0.94) \\
1.32(0.42)\end{array}$ & $\begin{array}{l}<0.001 \\
0.006\end{array}$ \\
\hline $\begin{array}{l}\text { Resident-level }{ }^{c} \\
\text { Resident characteristics } \\
\text { Gender (ref.: female) } \\
\text { Age } \\
\text { Mini-Mental State Examination }\end{array}$ & & & $\begin{array}{l}-0.09(0.40) \\
0.02(0.02) \\
0.02(0.03)\end{array}$ & $\begin{array}{l}0.821 \\
0.319 \\
0.563\end{array}$ \\
\hline $\begin{array}{l}\text { Diagnoses } \\
\text { Dementia (ref.: not present) } \\
\text { Depression (ref.: not present) } \\
\text { Musculoskeletal (ref.: not present) } \\
\text { Neuropathies (ref.: not present) } \\
\text { Tumor (ref.: not present) }\end{array}$ & & & $\begin{array}{l}-1.12(0.42) \\
-0.11(0.43) \\
0.46(0.37) \\
-0.39(0.58) \\
0.39(0.50)\end{array}$ & $\begin{array}{l}0.008 \\
0.807 \\
0.216 \\
0.502 \\
0.432\end{array}$ \\
\hline $\begin{array}{l}\text { Secondary outcomes } \\
\text { Geriatric Depression Scale } \\
\text { EQ-5D Quality of Life }\end{array}$ & & & $\begin{array}{l}0.08(0.06) \\
-0.07(0.01)\end{array}$ & $\begin{array}{l}0.139 \\
<0.001\end{array}$ \\
\hline $\begin{array}{l}\text { Nursing home-level }^{\mathrm{b}} \\
\text { Size in terms of resident care places } \\
\text { Number of registered practical nurses } \\
\text { Number of nursing assistants }\end{array}$ & & & $\begin{array}{l}0.01(0.01) \\
-0.07(0.03) \\
0.07(0.04)\end{array}$ & $\begin{array}{l}0.311 \\
0.076^{*} \\
0.148\end{array}$ \\
\hline $\begin{array}{l}\text { Random model part } \\
\text { Resident-level } \\
\text { Nursing home-level }\end{array}$ & $\begin{array}{l}13.42(0.91) \\
0.35(0.32)\end{array}$ & $\begin{array}{l}<0.001 \\
0.278\end{array}$ & $\begin{array}{l}9.90(0.78) \\
0.20(0.28)\end{array}$ & $\begin{array}{l}<0.001 \\
0.472\end{array}$ \\
\hline $\begin{array}{l}\text { ICC } \\
-2 \mathrm{LL} \\
\text { Sample size, nursing homes } \\
\text { Sample size, residents }\end{array}$ & $\begin{array}{l}0.0254 \\
2448.46 \\
15 \\
449\end{array}$ & & $\begin{array}{l}0.0300 \\
1811.96 \\
15 \\
347\end{array}$ & \\
\hline
\end{tabular}

Notes: ${ }^{a}$ Restricted maximum likelihood method, ${ }^{b}$ Fixed effects estimates: increase/decrease of maximum pain if explanatory variable increases by one unit, ${ }^{\mathrm{C}} \mathrm{Grand}$ mean centering, ${ }^{\mathrm{R}}$ Random effects estimates: unexplained variance components; ${ }^{*} \mathrm{p}<0$. I0. Bold font indicates statistical significance $(p<0.05)$.

Abbreviations: SE, standard error; $\mathrm{P}, \mathrm{P}$-value; ref, reference category; ICC, intraclass correlation coefficient; $-2 \mathrm{LL}$, log-likelihood ratio. 


\section{Residents with Severe Cognitive Impairment}

Multilevel logistic regression modelling results predicting proportions with PAINAD above cut-off in NHR with severe CI are displayed in Table 3. The enrolment of nursing homes to the intervention group had no significant effect $(\mathrm{OR}=0.89, \mathrm{p}=0.724)$ on PAINAD outcome neither in the adjusted final model $(n=222)$, nor in unadjusted models. Only one lower-level and one higher-level factor were associated with PAINAD scores above cut-off ( $\geq 2$ points). Presence of a probable pain indication was significantly predicted by decreasing abilities in cognitive functions according to MMSE (OR=0.92, CI 95\%=0.86-0.99, $\mathrm{p}=0.034)$; hence, NHR with higher MMSE scores were less likely to exhibit relevant signs of pain. Nursing homelevel variable "number of registered practical nurses" was found to affect the chances of pain in NHR with severe $\mathrm{CI}$-higher numbers of registered nurses were associated with significantly lower chances for NHR being in pain $(\mathrm{OR}=0.94$, CI 95\%=0.89-0.99, $\mathrm{p}=0.049)$. ICC was classified as rather low only in the final full model ( $\mathrm{ICC}_{\text {fullmodel }}$ $=4.3 \%$, but otherwise moderate $\left(\mathrm{ICC}_{\min }=7.6 \%\right.$, $\left.\mathrm{ICC}_{\max }=12.0 \%\right)$.

\section{Discussion}

This is one of the few studies to analyze the impact of nursing-related educational interventions on the pain situation of NHR with different levels of cognitive decline in a cluster randomized controlled trial. It was anticipated that the interventions within the scope of the project would result in changes on a structural level leading to

Table 3 Multilevel Logistic Regression Model Predicting PAINAD Above Cut-off (MMSE 0-9)

\begin{tabular}{|c|c|c|c|c|}
\hline \multirow{2}{*}{$\begin{array}{l}\text { Random Intercept Multilevel Logistic Regression } \\
\text { Model }^{\mathrm{a}} \text { for PAINAD }\end{array}$} & \multicolumn{2}{|c|}{ Null Model } & \multicolumn{2}{|c|}{ Resident and Nursing Home-Level Model } \\
\hline & OR $[\mathrm{Cl}]$ & $\mathbf{p}$ & OR $[\mathrm{Cl}]$ & p \\
\hline $\begin{array}{l}\text { Fixed model part } \\
\text { Intercept }^{\mathrm{b}} \\
\text { Time } \times \text { group }\end{array}$ & $0.33(0.22)$ & 0.129 & $\begin{array}{l}2.01(1.86) \\
0.89[0.46-1.72]\end{array}$ & $\begin{array}{l}0.281 \\
0.724\end{array}$ \\
\hline $\begin{array}{l}\text { Resident-level } \\
\text { Resident characteristics } \\
\text { Gender (ref.: female) } \\
\text { Age } \\
\text { Mini-Mental State Examination }\end{array}$ & & & $\begin{array}{l}0.77[0.44-\mathrm{I} .35] \\
\mathrm{I} .0 \mathrm{I}[0.96-\mathrm{I} .05] \\
\mathbf{0 . 9 2}[\mathbf{0 . 8 6}-\mathbf{0 . 9 9}]\end{array}$ & $\begin{array}{l}0.360 \\
0.885 \\
\mathbf{0 . 0 3 4}\end{array}$ \\
\hline $\begin{array}{l}\text { Diagnoses } \\
\text { Dementia (ref.: not present) } \\
\text { Depression (ref.: not present) } \\
\text { Musculoskeletal (ref.: not present) } \\
\text { Neuropathies (ref.: not present) } \\
\text { Tumor (ref.: not present) }\end{array}$ & & & $\begin{array}{l}0.76[0.46-1.27] \\
1.34[0.64-2.82] \\
1.08[0.58-1.99] \\
1.55[0.28-8.56] \\
1.29[0.69-2.43]\end{array}$ & $\begin{array}{l}0.292 \\
0.443 \\
0.814 \\
0.612 \\
0.417\end{array}$ \\
\hline $\begin{array}{l}\text { Secondary outcomes } \\
\text { Neuropsychiatric Inventory Index }\end{array}$ & & & $1.06[0.91-1.23]$ & 0.440 \\
\hline $\begin{array}{l}\text { Nursing home-level } \\
\text { Size in terms of resident care places } \\
\text { Number of registered practical nurses } \\
\text { Number of nursing assistants } \\
\text { Random model part } \\
\text { Nursing home-level }^{\text {b }}\end{array}$ & $0.45(0.30)$ & 0.136 & $\begin{array}{l}0.99[0.98-1.01] \\
0.94[0.89-0.99] \\
1.05[0.99-1.12] \\
0.15(0.24)\end{array}$ & $\begin{array}{l}0.440 \\
\mathbf{0 . 0 4 9} \\
0.123 \\
0.545\end{array}$ \\
\hline $\begin{array}{l}\mathrm{ICC}^{\mathrm{C}} \\
-2 \mathrm{LL} \\
\text { Sample size, nursing homes } \\
\text { Sample size, residents }\end{array}$ & $\begin{array}{l}0.1201 \\
1155.68 \\
15 \\
268\end{array}$ & & $\begin{array}{l}0.0430 \\
1008.21 \\
15 \\
222\end{array}$ & \\
\hline
\end{tabular}

Notes: ${ }^{a}$ Robust covariance method; baverage log-odds (SE, standard error); ' latent variable approach. Bold font indicates statistical significance ( $\left.p<0.05\right)$.

Abbreviations: PAINAD, Pain Assessment in Advanced Dementia; OR, odds ratio; $\mathrm{Cl} 95 \%$, confidence interval; $\mathrm{p}$, $\mathrm{p}$-value; ref, reference; ICC, intraclass correlation coefficient; -2LL, log-likelihood ratio. 
observable individual effects regarding the residents' pain situation. In particular, qualification and advanced training (ie pain nurses and pain care assistants) were assumed to extend the competencies of all nurses with different qualification levels directly involved in the nursing process, and thus, further increase the quality of the implemented pain management. However, the cRCT "PIASMA" showed no significant intervention effects in terms of reduced pain intensity scores in NHR with up to moderate cognitive impairment (MMSE 10-30, self-report) or treatment-relevant signs of pain in NHR with severe cognitive impairment (MMSE 0-9, proxy assessment).

Contrary to our hypothesis, the self-reported maximum pain intensity in NHR with no, mild or moderate cognitive impairment was even significantly higher in the intervention than in the control group at follow-up. To our knowledge, such opposite effects have not been reported in comparable broad-scale education intervention studies. We interpret these findings in terms of an "awareness effect" both on the part of nurses and residents. The provided education program aimed to train nurses comprehensively in order to enable pain nurses and pain care assistants to act as multiplicators and enhance knowledge transfer of relevant domains of pain management to the respective nursing teams at site. ${ }^{56}$ The web-based training as well as the discussion in the quality circles specifically focusing on pain assessment may have contributed to a topical sensitization on the subject of pain in general. As a consequence, pain was given more attention and was assessed more frequently in everyday care, thus, encouraging residents to elaborate their pain situation - the raised awareness may also have led to loosening reservations against reporting high pain intensities. Although all domains of pain management according to the relevant German Expert Standard for Chronic Pain Management in Nursing have been addressed, we assume that primarily the instructions regarding pain assessment had sustainable influences on care practices. ${ }^{41}$ Another recommendation of the implemented quality circles related to enable nurses to communicate suspicious residents' pain situations within the nursing team. Decisions of individual pain treatments should then rely on interdisciplinary discussions of such case reports. Especially such interdisciplinary discussions may have led to long-term changes in pain treatment. Similar procedures as part of a stepwise protocol for treating pain in NHR have been reported to successfully reduce pain intensity in a study by Sandvik et al. ${ }^{57}$ Although structured approaches are likely to facilitate multidisciplinary pain management, other studies reported no impact on the reduction of pain scores. ${ }^{58}$
While age, gender and cognitive status of NHR did not correlate with pain intensity scores, higher pain was reported from those NHR without a dementia diagnosis. This adds to the somewhat inconsistent empirical evidence examining the influence of cognition and dementia symptoms on self-reported pain. While Torvik et $\mathrm{al}^{59}$ found that pain was reported more frequently by residents with less cognitive impairments, another study ${ }^{60}$ observed the opposite effect. This may be moderated by a combined effect of residents' cognition-sensitive pain perceptions, nurses' presumptions about typical consequences of dementia and also the complexity to decide if self-reported information is still reliable or not: Prior work has highlighted the importance of assessing verbal and behavioral expressions simultaneously to self-report not only in NHR with more severe decline in cognitive function, but also in those with intermediate stages of dementia. ${ }^{34,61}$ The recognition of subtle changes in communication abilities from mild to moderate cognitive impairment in NHR is a challenging task for nurses and health-care professionals. Nurses are likely to assume that NHR lacking a dementia diagnosis can self-report on pain without any problems. At the same time, the transition from reliably communicating pain to not being able to self-report is fluid. This may have significant consequences for the provided care service as a dementia diagnosis might be seen as an obvious and "easy-to-decide" reason to give special (pain management) attention to such NHR. Reflecting on our findings, the question arises whether nurses are influenced in their care service provision depending on whether or not dementia diagnoses are documented. Paradoxically, this may lead to an under-treatment of pain in those NHR with up to moderate cognitive decline but without documented dementia. Our results illustrate the challenge in the process of nurses' pain identification in people with dementia and may add to the work of Chang et al, ${ }^{62}$ where interpersonal sensitivity and further social aspects were identified as crucial components to be considered in the process of nurses' pain identification. Nurses rely to some part on intuitive knowledge as pattern of personal knowledge, which is likely to be influenced by residents' health state and further documented cues. To overcome the described challenge, it seems necessary to attain a clear conceptualization for the identification of pain and to implement coordinated pain management processes in nursing homes.

For NHR with severe cognitive impairment, proportions of NHR with pain above PAINAD cut-off did not change due to intervention but remained basically the 
same. This is in line with another study about the impact of specific pain assessment interventions on pain scores in NHR with severe dementia. ${ }^{36}$ Although a special focus was set on assessing pain in NHR with severe cognitive impairment, the sole knowledge about pain assessment might not have an impact on further appropriate steps in pain management and our training videos for the nursing staff may have increased awareness but may not be able to change nurses' behavior to a significant extent. ${ }^{30,63}$ A recent European survey on pain assessment in older adults with dementia provides further insights into perceived challenges in pain management for persons with severe cognitive impairment. ${ }^{64}$ Health-care professionals stated that behavioral assessment instruments are often too difficult to complete due to the lack of objectivity. The main criticism was the high subjectivity in the evaluation of pain items (ie behavioral characteristics) and the difficulty to interpret resulting scores of the measures currently available - the authors conclude the high-priority importance to improve the usefulness of proxy pain assessment instruments. Our findings show that a more severe decline in cognitive function predicts higher chances to exhibit treatment-relevant signs of pain. We think that this resembles previous empirical evidence that NHR with severe cognitive decline are especially at risk to experience overlooked, under-treated, and maltreated pain. ${ }^{3,65}$ Proxy assessment of pain is based on the observation of behavioral signs. It may be the case that nurses judge behavioral signs of residents rather as reactions to unmet needs in the context of challenging behavior but less as possible consequences of NHR' pain. Former studies have shown that pharmacological and non-pharmacological pain therapy significantly reduces challenging behavior in NHR, therefore, indicating a non-ignorable relationship between pain and challenging behavior. ${ }^{66}$

On the nursing home-level, results indicate that lower self-reported maximum pain intensity is correlated with increasing numbers of registered nurses. This association was also observed in the subsample of residents not able to self-report, whereas the number of registered nurses significantly lessens the chances to be in treatment-relevant pain conditions. Similar positive relationships have been reported by a large number of studies examining nurse staffing and its consequences for patient outcomes in acute medical care settings. ${ }^{67}$ Positive effects of qualified nurses on care quality have also been reported for longterm care settings. ${ }^{68-70}$ Reflecting this evidence and in compliance with the claims of relevant German guidelines and standards, ${ }^{41,71}$ we cautiously interpret our findings in the way that registered nurses have a clearer conceptualization and more comprehensive understanding of pain management-without neglecting the essential contribution of nursing assistants and additional staff. These preliminary results seem to support the need for large-scale staff education and training in pain management of the nursing team including registered nurses and unregistered staff in order to achieve sustainable improvements of pain outcomes and associated phenomena in $\mathrm{NH}^{56}$

\section{Limitations}

Some limitations have to be acknowledged including the following: Inclusion of older adults into randomized controlled trials and conducting intervention studies in nursing homes is generally considered as complex. ${ }^{72-74}$ We faced several challenges during the study. Although deliberately conducting the study in nursing homes from only one single nursing home operator, access to the nursing homes, support during fieldwork and staffs' motivation to realize the intervention package varied vastly between the nursing homes. During the study fieldwork, considerable staff turnover at all hierarchy levels and superordinate organizational changes took place, possibly hindering a systematic penetration of our intervention. These processes may have also affected recruitment and participation of residents. While we have no explicit reason to assume that our observed population is not typical of that of German NH in general, we cannot rule out the possibility of sampling and coverage bias. Furthermore, measuring pain is always a compromise and potentially biased by the researchers' intention to transform individuals' subjective experiences into standardized and objectified scores. Besides, which pain outcomes for nursing home residents is best to use in terms of comprehensibility, usability, psychometric properties or sensitivity and specificity is debatable. Additional limitations relate to distinct features of the applied study design and rationale. Interventions focusing on changes in behavior and care procedures may take a considerable amount of time to be implemented and to show measurable effects-a necessary maturation of the implemented interventions may not have been realized in our 12-month intervention phase. Although the research team accompanied the interventions throughout the whole project, distinctive efforts to strictly control the intervention at nursing home sites might have fostered the effectivity of the intervention - an explicit framework for development and evaluation of complex interventions was 
not applied. Some statistical limitations should be considered. The final sample sizes were slightly lower as compared to the a priori calculated sample sizes. While the sample sizes within each cluster are more than sufficient, the rather low number of 15 clusters may be critical in multilevel modelling. Such a small sample size may bias estimates of higher-level predictors not in terms of coefficients but in terms of standard errors, thus risking type-1 error inflation. ${ }^{75}$ Therefore, our results regarding the significant effects of the number of registered nurses (higherlevel effect) should be treated with caution. However, the individual-level predictors are hardly at risk to be biased. To check on this, sensitivity analysis using fixed effect models eliminating between-cluster variation introducing nursing home dummies were performed (not presented here; available upon request). These additional analyses demonstrated comparable results regarding the residentlevel predictors. In the sample of NHR $\mathrm{MMSE}_{10-30}$, slightly higher standard errors were observed leading to a significant by trend $(p<0.10)$ intervention effect but replicated the main effects' direction. Additionally, multilevel analyses were performed following a strict intentionto-treat principle. The respected nursing home, where no interventions were implemented, was allocated to the intervention group (as randomized) in these sensitivity analyses (not presented here; available upon request). The studies' findings regarding the intervention effects did not differ from the presented results. Finally, additional subgroup analyses (ie multilevel models separated by residents with no, mild, moderate cognitive impairment) were not feasible due to limited subsample sizes.

\section{Conclusion}

This cluster-randomized controlled trial did not demonstrate an improvement of resident-specific pain outcomes due to the implementation of nursing-related educational interventions. No significant reductions of pain intensity or treatment-relevant pain were found after intervention. This adds to the mixed and controversial empirical evidence regarding the effectiveness of such large-scale education interventions. However, significant predictors of maximum pain intensity and treatment-relevant behavioral signs of pain at the resident (dementia and cognitive impairment, quality of life) and nursing-home level (number of registered nurses) were reported. These findings may also contribute to the planning of future studies in terms of study design, decision on outcomes or focus of intended interventions. Selected findings suggest nurses' raised awareness towards pain management but overall results indicate that large-scale educational interventions may be less effective in complex nursing home settings without a systematic assessment of change management possibilities at a structural NH-level as well as specific individual-based intervention strategies. Still, we assume that the applied intervention increased nurses' skills, knowledge and process management in pain management on site and leads to ongoing improvements regarding the residents' pain situation. In order to ensure a sustainable implementation of pain management in long-term care, future studies should not solely rely on knowledge and training interventions but should combine such efforts with concrete individualspecific interventions or supervised and controlled implementation of stepwise-protocols. As further measures to achieve a high-quality care of pain, internal audits in terms of case-wise document analysis, case reports and regular interdisciplinary quality circles are still recommended. Also, the systematic evaluation of organizational structures and existing written procedures should be part of future studies. The identification of instruments to evaluate the effectiveness of education and teaching programs as part of nursing curriculums as well as to evaluate skills and competencies in nursing practice should be given highpriority in future research. Finally, our non-significant findings do not necessarily propose a general insufficiency of large-scale educational intervention packages, but may also be attributed to the above discussed limitations of our study.

\section{Data Sharing Statement}

A minimum data set is available from the corresponding author upon reasonable request.

\section{Acknowledgments}

We would like to thank 'Smart q' (https://www.smart-q.de/), who provided the IT structure for data collection and archiving, Firuzan Sari Kundt, who provided English language editing, all the nursing home staff, all the interviewers, and most sincerely thank all the participating nursing home residents, their relatives, and legal guardians.

\section{Disclosure}

The trial was funded by the nursing home operator Curanum AG and the province of Salzburg, Austria. J Osterbrink reports grants from Curanum AG and Province of Salzburg during the conduct of the study. I Gnass reports grants from nursing home operator Curanum AG and grants from province of Salzburg, Austria, outside the submitted work. The 
authors declare that they have no other competing interests or conflicts of interest in this work. The nursing home operator did not influence any phases of the project, neither during writing the study protocol, nor while conducting the study, intervention planning or analyses and interpretation of the results.

\section{References}

1. Kalinowski S, Budnick A, Kuhnert R, et al. Nonpharmacologic pain management interventions in German nursing homes: a cluster randomized trial. Pain Manag Nurs. 2015;16(4):464-474. doi:10.1016/j. pmn.2014.09.002

2. Lukas A, Mayer B, Onder G, Bernabei R, Denkinger MD. Pain therapy in German long-term care facilities in a European comparison. Results of the SHELTER study. Schmerz. 2015;29(4):411-421. doi:10.1007/s00482-015-0004-6

3. Cravello L, Di Santo S, Varrassi G, et al. Chronic pain in the elderly with cognitive decline: a narrative review. Pain Ther. 2019;8 (1):53-65. doi:10.1007/s40122-019-0111-7

4. Takai Y, Yamamoto-Mitani N, Okamoto Y, Koyama K, Honda A. Literature review of pain prevalence among older residents of nursing homes. Pain Manage Nurs. 2010;11(4):209-223. doi:10.1016/j. pmn.2010.08.006

5. Achterberg WP, Gambassi G, Finne-Soveri H, et al. Pain in European long-term care facilities: cross-national study in Finland, Italy and the Netherlands. Pain. 2010;148(1):70-74. doi:10.1016/j.pain.2009.10.008

6. Osterbrink J, Hufnagel M, Kutschar P, et al. The pain situation for residents of nursing homes. Results of a study in Munster. Schmerz. 2012;26(1):27-35. doi:10.1007/s00482-011-1127-z

7. Auer SR, Hofler M, Linsmayer E, et al. Cross-sectional study of prevalence of dementia, behavioural symptoms, mobility, pain and other health parameters in nursing homes in Austria and the Czech Republic: results from the DEMDATA project. BMC Geriatr. 2018;18(1):178. doi:10.1186/s12877-018-0870-8

8. Herr K. Pain assessment strategies in older patients. J Pain. 2011;12 (3, Suppl 1):S3-S13. doi:10.1016/j.jpain.2010.11.011

9. van Dalen-kok AH, Pieper MJ, de Waal MW, Lukas A, Husebo BS, Achterberg WP. Association between pain, neuropsychiatric symptoms, and physical function in dementia: a systematic review and meta-analysis. BMC Geriatr. 2015;15(1):49. doi:10.1186/s12877-015-0048-6

10. Husebo B, Kunz M, Achterberg WP, et al. Pain Assessment and treatment. challenges in patients with dementia. Zeitschrift für Neuropsychologie. 2012;23(4):237-246. doi:10.1024/1016-264X/a000078

11. Klapwijk MS, Caljouw MAA, Pieper MJC, Putter H, van der Steen JT, Achterberg WP. Change in quality of life after a multidisciplinary intervention for people with dementia: a cluster randomized controlled trial. Int $J$ Geriatr Psychiatry. 2018;33 (9):1213-1219. doi:10.1002/gps.v33.9

12. Erdal A, Flo E, Selbaek G, et al. Associations between pain and depression in nursing home patients at different stages of dementia. $J$ Affect Disord. 2017;218:8-14. doi:10.1016/j.jad.2017.04.038

13. Hoffmann F, Kaduszkiewicz H, Glaeske G, van den Bussche H, Koller D. Prevalence of dementia in nursing home and community-dwelling older adults in Germany. Aging Clin Exp Res. 2014;26(5):555-559. doi:10.10 07/s40520-014-0210-6

14. Bisla J, Calem M, Begum A, Stewart R. Have we forgotten about dementia in care homes? The importance of maintaining survey research in this sector. Age Ageing. 2011;40(1):5-6. doi:10.1093/ageing/afq150

15. Horgas AL, Nichols AL, Schapson CA, Vietes K. Assessing pain in persons with dementia: relationships among the non-communicative patient's pain assessment instrument, self-report, and behavioral observations. Pain Manag Nurs. 2007;8(2):77-85. doi:10.1016/j. pmn.2007.03.003
16. Hadjistavropoulos T, Fitzgerald TD, Marchildon GP. Practice guidelines for assessing pain in older persons with dementia residing in long-term care facilities. Physiother Can Spr. 2010;62(2):104-113. doi:10.3138/physio.62.2.104

17. Pautex S, Michon A, Guedira M, et al. Pain in severe dementia: self-assessment or observational scales? J Am Geriatr Soc. 2006;54 (7):1040-1045. doi:10.1111/jgs.2006.54.issue-7

18. Herr K, Coyne PJ, McCaffery M, Manworren R, Merkel S. Pain assessment in the patient unable to self-report: position statement with clinical practice recommendations. Pain Manag Nurs. 2011;12 (4):230-250. doi:10.1016/j.pmn.2011.10.002

19. Clark P, Tucke SS, Whitlatch CJ. Consistency of information from persons with dementia: an analysis of differences by question type. Dementia. 2008;7(3):341-358. doi:10.1177/1471301208093288

20. Whitlatch CJ, Feinberg LF, Tucke S. Accuracy and consistency of responses from persons with cognitive impairment. Dementia. 2005;4 (2):171-183. doi:10.1177/1471301205051091

21. Lukas A, Niederecker T, Günther I, Mayer B, Nikolaus T. Self- and proxy report for the assessment of pain in patients with and without cognitive impairment. Experiences gained in a geriatric hospital. Zeitschrift für Gerontologie und Geriatrie. 2013;214-221. doi:10.1007/s00391-0130475-y

22. Ballard C, Smith J, Husebo B, Aarsland D, Corbett A. The role of pain treatment in managing the behavioural and psychological symptoms of dementia (BPSD). Int J Palliat Nurs. 2011;17(9):420,422, 424. doi:10.12968/ijpn.2011.17.9.420

23. Reynolds KS, Hanson LC, DeVellis RF, Henderson M, Steinhauser KE. Disparities in pain management between cognitively intact and cognitively impaired nursing home residents. J Pain Symptom Manage. 2008;35(4):388-396. doi:10.1016/j.jpainsymman.2008.01.001

24. Hemmingsson ES, Gustafsson M, Isaksson U, et al. Prevalence of pain and pharmacological pain treatment among old people in nursing homes in 2007 and 2013. Eur J Clin Pharmacol. 2018;74 (4):483-488. doi:10.1007/s00228-017-2384-2

25. Miu DKY, Chan KC. Under-detection of pain in elderly nursing home residents with moderate to severe dementia. $J$ Clin Gerontol Geriatrics. 2014;5(1):23-27. doi:10.1016/j.jcgg.2013.09.001

26. Schreier MM, Stering U, Pitzer S, Iglseder B, Osterbrink J. Pain and pain-assessment in nursing homes. Results of the OSiA study. Schmerz. 2015;29(2):203-210. doi:10.1007/s00482-014-1509-0

27. Swafford KL, Miller LL, Tsai PF, Herr KA, Ersek M. Improving the process of pain care in nursing homes: a literature synthesis. $J \mathrm{Am}$ Geriatr Soc. 2009;57(6):1080-1087. doi:10.1111/j.1532-5415. 2009.02274.x

28. Hines S, Ramsbotham J, Coyer F. Interventions for improving the research literacy of nurses: a systematic review. JBI Database Syst Rev Implementation Rep. 2016;14(2):256-294. doi:10.11124/jbisrir2016-2378

29. Fuchs-Lacelle S, Hadjistavropoulos T, Lix L. Pain assessment as intervention - A study of older adults with severe dementia. Clin $J$ Pain. 2008;24(8):697-707. doi:10.1097/AJP.0b013e318172625a

30. Gagnon MM, Hadjistavropoulos T, Williams J. Development and mixed-methods evaluation of a pain assessment video training program for long-term care staff. Pain Res Manag. 2013;18(6):307-312. doi:10.1155/2013/659320

31. Ospina MB, Taenzer P, Rashiq S, et al. A systematic review of the effectiveness of knowledge translation interventions for chronic noncancer pain management. Pain Res Manag. 2013;18(6):e129-e141. doi: $10.1155 / 2013 / 120784$

32. Husebo B, Ballard C, Aarsland D. Pain treatment of agitation in patients with dementia: a systematic review. Int J Geriatr Psychiatry. 2011;26 (10):1012-1018. doi:10.1002/gps.2649

33. Pieper MJ, Francke AL, van der Steen JT, et al. Effects of a stepwise multidisciplinary intervention for challenging behavior in advanced dementia: a cluster randomized controlled trial. J Am Geriatr Soc. 2016;64(2):261-269. doi:10.1111/jgs.2016.64.issue-2 
34. Hadjistavropoulos T, Kaasalainen S, Williams J, Zacharias R Improving pain assessment practices and outcomes in long-term care facilities: a mixed methods investigation. Pain Manag Nurs. 2014;15(4):748-759. doi:10.1016/j.pmn.2013.07.009

35. Gunnarsdottir S, Zoega S, Serlin RC, et al. The effectiveness of the pain resource nurse program to improve pain management in the hospital setting: a cluster randomized controlled trial. Int $J$ Nurs Stud. 2017;75:83-90. doi:10.1016/j.ijnurstu.2017.07.009

36. Rostad HM, Utne I, Grov EK, Smastuen MC, Puts M, Halvorsrud L. The impact of a pain assessment intervention on pain score and analgesic use in older nursing home residents with severe dementia: a cluster randomised controlled trial. Int J Nurs Stud. 2018;84:52-60. doi:10.1016/j.ijnurstu.2018.04.017

37. Guion V, De Souto Barreto P, Sourdet S, Rolland Y. Effect of an educational and organizational intervention on pain in nursing home residents: a nonrandomized controlled trial. J Am Med Dir Assoc. 2018;19(12):1118-1123.e1112. doi:10.1016/j.jamda.2018.09.031

38. Knopp-Sihota JA, Patel P, Estabrooks CA. Interventions for the treatment of pain in nursing home residents: a systematic review and meta-analysis. J Am Med Dir Assoc. 2016;17(12):1163e11191163 e1128. doi:10.1016/j.jamda.2016.09.016

39. Corbett A, Husebo BS, Achterberg WP, Aarsland D, Erdal A, Flo E. The importance of pain management in older people with dementia. Br Med Bull. 2014;111(1):139-148. doi:10.1093/bmb/ldu023

40. Deutsche Schmerzgesellschaft e.V. (German Pain Society - Chapter of the International Association for the Study of Pain 'IASP') Schmerztherapeutisches Curriculum für die integrierte Aus-, Weiterund Fortbildung in der Pflege [German] [Curriculum for the integrated training, continuing and advanced education in pain therapy]. 2014, 2018, 2019. Available from: https://www.dgss.org/fileadmin/pdf/curriculum pflege_aktuell_maerz_2019.doc_2.pdf. Accessed September.14, 2019.

41. DNQP. Expert Standard for Chronic Pain Management in Nursing. Osnabrück, Germany: German Network for Quality Development in Nursing; 2015.

42. Folstein MF, Folstein SE, McHugh PR. "Mini-Mental State". A practical method for grading the cognitive state of patients for the clinician. J Psychiatr Res. 1975;12(3):189-198. doi:10.1016/ 0022-3956(75)90026-6

43. Folstein MF, Folstein SE, Fanjiang G. MMSE Mini-Mental State Examination Clinical Guide. Odessa: Psychological Assessment Resources; 2001.

44. Whitlatch CJ, Menne HL. Don't forget about me! Decision making by people with dementia. Generations - J Am Soc Aging. 2009;33(1):66-73.

45. Budnick A, Kuhnert R, Könner F, Kalinowski S, Kreutz R, Dräger D. Validation of a modified German version of the brief pain inventory for use in nursing home residents with chronic pain. J Pain. 2016;17 (2):248-256. doi:10.1016/j.jpain.2015.10.016

46. Cleeland C. Pain and its severity in cancer patients. PRN Forum. 1982;1(2):1-2.

47. Warden V, Hurley AC, Volicer L. Development and psychometric evaluation of the Pain Assessment in Advanced Dementia (PAINAD) scale. J Am Med Dir Assoc. 2003;4(1):9-15. doi:10.1097/01.JAM.00 00043422.31640.F7

48. Basler HD, Huger D, Kunz R, et al. Beurteilung von Schmerz bei Demenz (BESD). Untersuchung zur Validität eines Verfahrens zur Beobachtung des Schmerzverhaltens [Assessment of pain in advanced dementia. Construct validity of the German PAINAD]. Schmerz. 2006;20(6):519-526. German. doi:10.1007/s00482-0060490-7

49. Zwakhalen S, Van der Steen JT, Najim MD. Which score represents pain on the observational PAINAD pain scale for dementia patient'? Int Psychogeriatrics. 2011;23:48-49.

50. Allgaier AK, Kramer D, Mergl R, Fejtkova S, Hegerl U. Validity of the geriatric depression scale in nursing home residents: comparison of GDS-15, GDS-8, and GDS-4. Psychiat Prax. 2011;38(6):280-286. doi:10.1055/s-0030-1266105
51. Yesavage JA, Brink TL, Rose TL, et al. Development and validation of a geriatric depression screening scale - a preliminary-report. $J$ Psychiat Res. 1983;17(1):37-49. doi:10.1016/0022-3956(82)90033-4

52. The EuroQol Group. EuroQol-a new facility for the measurement of health-related quality of life. Health Policy. 1990;16(3):199-208. doi:10.1016/0168-8510(90)90421-9

53. Hinz A, Klaiberg A, Brahler E, Konig HH. Der Lebensqualitätsfragebogen EQ-5D: Modelle und Normwerte für die Allgemeinbevölkerung [The Quality of Life Questionnaire EQ-5D: modelling and norm values for the general population]. Psychother Psychosom Med Psychol. 2006;56(2):42-48. German. doi:10.1055/ s-2005-867061

54. Zuidema SU, de Jonghe JF, Verhey FR, Koopmans RT. Environmental correlates of neuropsychiatric symptoms in nursing home patients with dementia. Int J Geriatr Psychiatry. 2010;25(1):14-22. doi:10.1002/ gps.2292

55. Dworkin RH, Turk DC, Farrar JT, et al. Core outcome measures for chronic pain clinical trials: IMMPACT recommendations. Pain. 2005;113(1-2):9-19. doi:10.1016/j.pain.2004.09.012

56. RCN (Royal College of Nursing). Pain knowledge and skills framework for the nursing team; 2015. Available from: https://www.brit ishpainsociety.org/static/uploads/resources/files/RCN_KSF_2015. pdf. Accessed May.29, 2019.

57. Sandvik RK, Selbaek G, Seifert R, et al. Impact of a stepwise protocol for treating pain on pain intensity in nursing home patients with dementia: a cluster randomized trial. Eur J Pain. 2014;18 (10):1490-1500. doi:10.1002/ejp.2014.18.issue-10

58. Jones KR, Fink R, Vojir C, et al. Translation research in long-term care: improving pain management in nursing homes. Worldviews Evidence-Based Nurs. 2004;1(Suppl 1):S13-S20. doi:10.1111/ j.1524-475X.2004.04045.x

59. Torvik K, Kaasa S, Kirkevold O, Rustoen T. Pain in patients living in Norwegian nursing homes. Palliat Med. 2009;23(1):8-16. doi:10.11 77/0269216308098800

60. Leong IY, Nuo TH. Prevalence of pain in nursing home residents with different cognitive and communicative abilities. Clin J Pain. 2007;23(2):119-127. doi:10.1097/01.ajp.0000210951.01503.3b

61. Hadjistavropoulos T, Craig KD. A theoretical framework for understanding self-report and observational measures of pain: a communications model. Behav Res Ther. 2002;40(5):551-570. doi:10.1016/S00057967(01)00072-9

62. Chang SO, Oh Y, Park EY, Kim GM, Kil SY. Concept analysis of nurses' identification of pain in demented patients in a nursing home: development of a hybrid model. Pain Manag Nurs. 2011;12 (2):61-69. doi:10.1016/j.pmn.2010.05.007

63. Chan SS, Leung DY, Leung AY, et al. A nurse-delivered brief health education intervention to improve pneumococcal vaccination rate among older patients with chronic diseases: a cluster randomized controlled trial. Int J Nurs Stud. 2015;52(1):317-324. doi:10.1016/j. ijnurstu.2014.06.008

64. Zwakhalen S, Docking RE, Gnass I, et al. Pain in older adults with dementia: a survey across Europe on current practices, use of assessment tools, guidelines and policies. Schmerz. 2018. doi:10.1007/ s00482-018-0290-x

65. Husebo B, Achterberg WP, Lobbezoo F, et al. Pain in patients with dementia: a review of pain assessment and treatment challenges. Nor Epidemiol. 2012;22(2):243-251.

66. Husebo B, Ballard C, Sandvik R, Nilsen OB, Aarsland D. Efficacy of treating pain to reduce behavioural disturbances in residents of nursing homes with dementia: cluster randomised clinical trial. $B M J$. 2011;343(jul15 1):d4065. doi:10.1136/bmj.d4065

67. West E, Barron DN, Harrison D, Rafferty AM, Rowan K, Sanderson C. Nurse staffing, medical staffing and mortality in intensive care: an observational study. Int J Nurs Stud. 2014;51 (5):781-794. doi:10.1016/j.ijnurstu.2014.02.007 
68. Castle NG, Anderson RA. Caregiver staffing in nursing homes and their influence on quality of care: using dynamic panel estimation methods. Med Care. 2011;49(6):545-552. doi:10.1097/MLR.0b0 $13 \mathrm{e} 31820 \mathrm{fbca} 9$

69. Shin JH. Nursing staff characteristics on resident outcomes in nursing homes. J Nurs Res. 2018;27(1):1.

70. Konetzka RT, Stearns SC, Park J. The staffing-outcomes relationship in nursing homes. Health Serv Res. 2008;43(3):1025-1042. doi:10.1111/ j.1475-6773.2007.00803.x

71. AWMF. S3-Leitlinie: Schmerzassessment bei älteren Menschen in der vollstationären Altenhilfe [S3-Guideline: pain assessment in older persons in residential care]; 2018. Available from: https://www.awmf.org/uploads/ tx_szleitlinien/145-001m_S3_Schmerzassessment-bei-aelterenMenschen_in-der-vollstationaeren_Altenhilfe_2018-02_01.pdf. Accessed January.15, 2019. German.
72. Zulman DM, Sussman JB, Chen X, Cigolle CT, Blaum CS, Hayward RA. Examining the evidence: a systematic review of the inclusion and analysis of older adults in randomized controlled trials. J Gen Intern Med. 2011;26 (7):783-790. doi:10.1007/s11606-010-1629-x

73. Hall S, Longhurst S, Higginson IJ. Challenges to conducting research with older people living in nursing homes. BMC Geriatr. 2009;9 (1):38-46. doi:10.1186/1471-2318-9-38

74. Fossey J, Garrod L, Lawrence V, Testad I, Stafford J, Murray J. "We should see her like part of the team": an investigation into care home staff's experiences of being part of an RCT of a complex psychosocial intervention. Aging Ment Health. 2018;24(1):178-185.

75. Maas ML, Hox J. Sufficient sample sizes for multilevel modeling. Methodology. 2005;1(3):86-92. doi:10.1027/1614-2241.1.3.86

\section{Publish your work in this journal}

The Journal of Pain Research is an international, peer reviewed, open access, online journal that welcomes laboratory and clinical findings in the fields of pain research and the prevention and management of pain. Original research, reviews, symposium reports, hypothesis formation and commentaries are all considered for publication. The manuscript management system is completely online and includes a very quick and fair peer-review system, which is all easy to use. Visit http:// www.dovepress.com/testimonials.php to read real quotes from published authors. 